\title{
Banff lesions and renal allograft survival in chronic-active antibody mediated rejection
}

\author{
Kasia A. Sablik ${ }^{\mathrm{a}}$, Marian C. Clahsen- van Groningen ${ }^{\mathrm{b}}$, Jeffrey Damman ${ }^{\mathrm{b}}$, Dave L. Roelen ${ }^{\mathrm{c}}$, \\ Michiel G.H. Betjes ${ }^{\mathrm{a},}$ \\ a Department of Nephrology and Transplantation, Erasmus Medical Center, Rotterdam, the Netherlands \\ ${ }^{\mathrm{b}}$ Department of Pathology, Erasmus Medical Center, Rotterdam, the Netherlands \\ ${ }^{\mathrm{c}}$ Department of Immunohematology and Blood Transfusion, Leiden University Medical Center, Leiden, the Netherlands
}

\section{A R T I C L E I N F O}

\section{Keywords:}

Kidney

Transplantation

Antibody-mediated rejection

Histopathology

Graft survival

\begin{abstract}
A B S T R A C T
Aims: Chronic-active antibody mediated rejection (c-aABMR) is a major cause of kidney graft loss. Currently, little is known about the relation between histopathologic parameters and renal allograft survival.

Methods and results: Between 2008 and 2014, 41 patients with a progressive decrease in renal function were diagnosed with c-aABMR according to Banff 2015 and followed up for at least 3 years. Clinical and renal biopsy characteristics were analyzed for association with graft survival.

During follow-up 26 cases lost their graft because of c-aABMR at a median follow up of 40 months after diagnosis.

Cases with v-lesions in their biopsy had a significant higher loss of eGFR prior to diagnosis. The total in flammation score $(r=-0.45 p=.007)$ and the severity of interstitial fibrosis $(r=-0.38 p=.023)$ were related to the eGFR at time of biopsy.

Univariate regression analysis showed that eGFR at time of biopsy, total inflammation, interstitial fibrosis and the sum chronicity score were significantly related to the risk for graft failure during follow-up. In a multivariate analysis only the severity of interstitial fibrosis remained associated with decreased graft survival (HR 1.9 per score point, $95 \%$ CI $1.2-2.8, p=.004$ )

Conclusion: Severity of renal interstitial fibrosis and not inflammation predicts graft survival in cases of caABMR.
\end{abstract}

\section{Introduction}

Although short-term results of kidney transplantation have improved significantly in the last decades, long-term renal allograft survival has shown little improvement [1,2]. Therefore, the causes that pose a barrier to long-term renal allograft survival need to be addressed in order to further the progress in maximizing the lifespan of transplanted kidneys [3-5].

A very important barrier, that has only been fully recognized in recent years, is chronic-active antibody-mediated rejection (c-aABMR). The clinical hallmark of c-aABMR is progressive loss of allograft function with nephrotic range proteinuria. Chronic-aABMR is now regarded as one of the most important contributors to late renal allograft failure $[6,7]$. The prognosis of c-aABMR is very poor with most patients reaching graft failure within 2-3 years after diagnosis [7-11]. Treatment of c-aABMR has become a major unmet need in transplant nephrology as there are currently no established effective treatment protocols available [12-14].

Surprisingly, it is largely unknown whether factors such as renal histomorphology or clinical parameters are associated with renal allograft survival in patients with c-aABMR. Identification of these key parameters could be of relevance for stratification of patients in future clinical trials or to decide whether or not to treat a patient with caABMR of the kidney allograft.

Previous studies have indicated that cases with a higher degree of microvascular inflammation may be associated with a better response to therapy and that a compound chronicity score in cases of transplant glomerulopathy predisposes for poorer allograft survival [15,16]. However, these results were not obtained in clearly Banff-defined cohorts of c-aABMR patients receiving an identical therapeutic intervention. In this study, the relation between histomorphologic lesions according to Banff 2015 criteria and graft survival were analyzed in a

\footnotetext{
* Corresponding author at: Erasmus Medical Center, Department of Nephrology \& Transplantation, Rg-527, P.O. Box 2040, 3000 CA Rotterdam, the Netherlands.

E-mail address: m.g.h.betjes@erasmusmc.nl (M.G.H. Betjes).
} 
well-defined group of patients with c-aABMR with a minimum follow up of 3 years or until graft failure was reached.

\section{Objective}

To define prognostic histopathology parameters for the risk of graft loss in cases with c-aABMR

\section{Material and methods}

\subsection{Study population}

We retrospectively included 41 renal transplant patients who had a biopsy proven diagnosis of c-aABMR between October 2008 and December 2014. No selection was made for inclusion in this study other than having a histologically proven diagnosis of c-aABMR. Time of last follow-up was December 2017. All patients underwent a for cause biopsy at least 1 year post transplantation due to a progressive decline in renal function. Renal biopsies were scored according to the Banff ' 15 criteria [17] by 2 independent experienced renal pathologists reaching consensus on the severity of the different lesions in all cases. In accordance with the Banff ' 15 criteria, the biopsies showing all histological criteria for c-aABMR but without detectable DSA were included though designated as suspicious for c-aABMR.

The Banff lesions could be fully assessed in 35 of 41 patients. Immunohistochemical staining for C4d was not available in 1 patient.

Following our local protocol, all patients with c-aABMR were administered three doses of $1 \mathrm{~g}$ intravenous MP over a 3 day period combined with a single dose of intravenous immunoglobulins $(1 \mathrm{~g} / \mathrm{kg}$ body weight) on the second day of treatment [18]. Renal allograft function was assessed on the basis of estimated glomerular filtration rate (eGFR in $\mathrm{mL} / \mathrm{min} / 1.73 \mathrm{~m}^{2}$ ). All eGFR measurements between one year prior to treatment and one year after treatment were included to calculate the decrease in eGFR within a given period of time. Cases included had at least 5 measurements of eGFR at regular intervals in the year before and after diagnosis of c-aABMR. Allograft failure was defined by the need for starting dialysis treatment or kidney retransplantation.

The local medical ethical committee approved the study (april 16th 2015;MEC-2015-222) and humans involved in this study were treated in a manner in accordance with the Declaration of Helsinki and the Declaration of Istanbul. All patients gave their written informed consent for participation in this study.

\subsection{Characterization of anti-HLA antibodies}

All patients were transplanted with a CDC negative crossmatch. All sera prior to treatment were screened for the presence of donor-specific antibodies against HLA (DSA). Patient serum samples were screened within a 3 month time period before or after diagnostic biopsy for the presence or absence of HLA antibodies using the Lifecodes Lifescreen Deluxe (LMX) kit, according to the manufacturer's manual (Immunocor Transplant Diagnostics Inc. Stamford, CT, USA). Samples were considered positive for either HLA class I (HLA-A or HLA-B or HLA-C) or HLA class II (HLA-DQ or HLA-DR) antibodies were further analyzed with a Luminex Single Antigen assay, using LABscreen HLA class I and class II antigen beads (One Lambda Canoga Park, GA, USA).

\subsection{Statistical analysis}

Normally distributed data are expressed as mean $+/-\mathrm{SD}$, nonnormally distributed data as median with range. A $p$-value of $<0.05$ was considered statistically significant.

Death-censored graft survival was assessed by Kaplan-Meier analysis with log-rank statistics for difference between strata. For this analysis the histomorphologic lesions were analyzed dichotomously (if
Table 1

Clinical and immunological characteristics of 41 cases of chronic-active antibody mediated rejection.

\begin{tabular}{ll}
\hline Age in years median (range) & $52(19-72)$ \\
\hline Gender, male \% & $63.4 \%$ \\
Donor type: & $29 \%$ \\
$\quad$ Deceased & $27 \%$ \\
$\quad$ Living related & $44 \%$ \\
$\quad$ Living unrelated & $41.5 \%$ \\
Previous transplantation \% & $76(12-218)$ \\
Time to diagnosis in months median (range) & $27(8-86)$ \\
Follow up time in months median (range) & $3(1-6)$ \\
Number of HLA mismatches, median (range) & $17(43.9 \%)$ \\
DSA present, n (\%) & \\
Type of DSA: & \\
$\quad$ Anti-HLA class 1 ( $n=3)$ & \\
$\quad$ Anti-HLA class 2 ( $n=15)$ & $26.8 \%$ \\
Previous biopsy proven acute rejection & $0.8(0.1-11.5)$ \\
Proteinuria (g/L) at time diagnosis, median (range) & \\
eGFR mL/min/1.73m ${ }^{2}$, median (range) & $37(15-78)$ \\
$\quad 1$ year before diagnosis & $30(15-45)$ \\
$\quad$ Time of diagnosis & $30(8-47)$ \\
$\quad 1$ year after diagnosis & \\
Immunosuppressive medication (\%) & 81 \\
Tacrolimus & 5 \\
Cyclosporine & 90 \\
Mycophenolate mofetil & 22 \\
Prednisone & 7 \\
Other & \\
\hline
\end{tabular}

possible, see results). Cases in which lesions were scored as minimal to mild (0-1) were compared to cases in which lesions were scored as moderate to severe (2-3). Both v-lesions and C4d staining were scored as either being present or absent.

Univariate and multivariate logistic regression analysis was performed to identify an association between histomorphological lesions (as continuous variables) and clinical variables with graft survival. Variables with a $p$-value of $<0.1$ were considered for a multivariate proportional hazard analysis in a forward stepwise manner. Statistical analysis was performed with software IBM SPSS statistics 21 .

\section{Results}

\subsection{Clinical characteristics}

The demographic and clinical characteristics at time of for cause biopsy are summarized in Table 1 . Time to biopsy from the moment of transplantation showed a median of 76 (12-218) months. The immunosuppressive regimen predominantly consisted of combined tacrolimus and mycophenolate mofetil (71\%). During the first year of follow up no deaths occurred but 3 patients progressed to allograft failure. A total of 26 cases lost their renal allograft as a result to caABMR at a median follow up of 40 months after diagnosis by renal biopsy.

Twenty-six percent of c-aABMR cases had a previous biopsy proven acute rejection (BPAR). The vast majority (85\%) of the BPAR was T-cell mediated rejection (TCMR) and occurred within the first months after transplantation. Proteinuria varied substantially from marginally present to several gram/L.

\subsection{Histomorphological characteristics}

The scores of the lesions of the renal biopsies $(n=35)$, graded by the Banff criteria, are shown in Fig. 1. In 6 cases there were an insufficient number of non-sclerotic glomeruli in the biopsy to fulfill the criteria for scoring according to the Banff classification.

All biopsies showed glomerular basement membrane double contours (cg) of which $>90 \%$ were scored as either $\operatorname{cg} 2$ or $\mathrm{cg} 3$. In addition, 


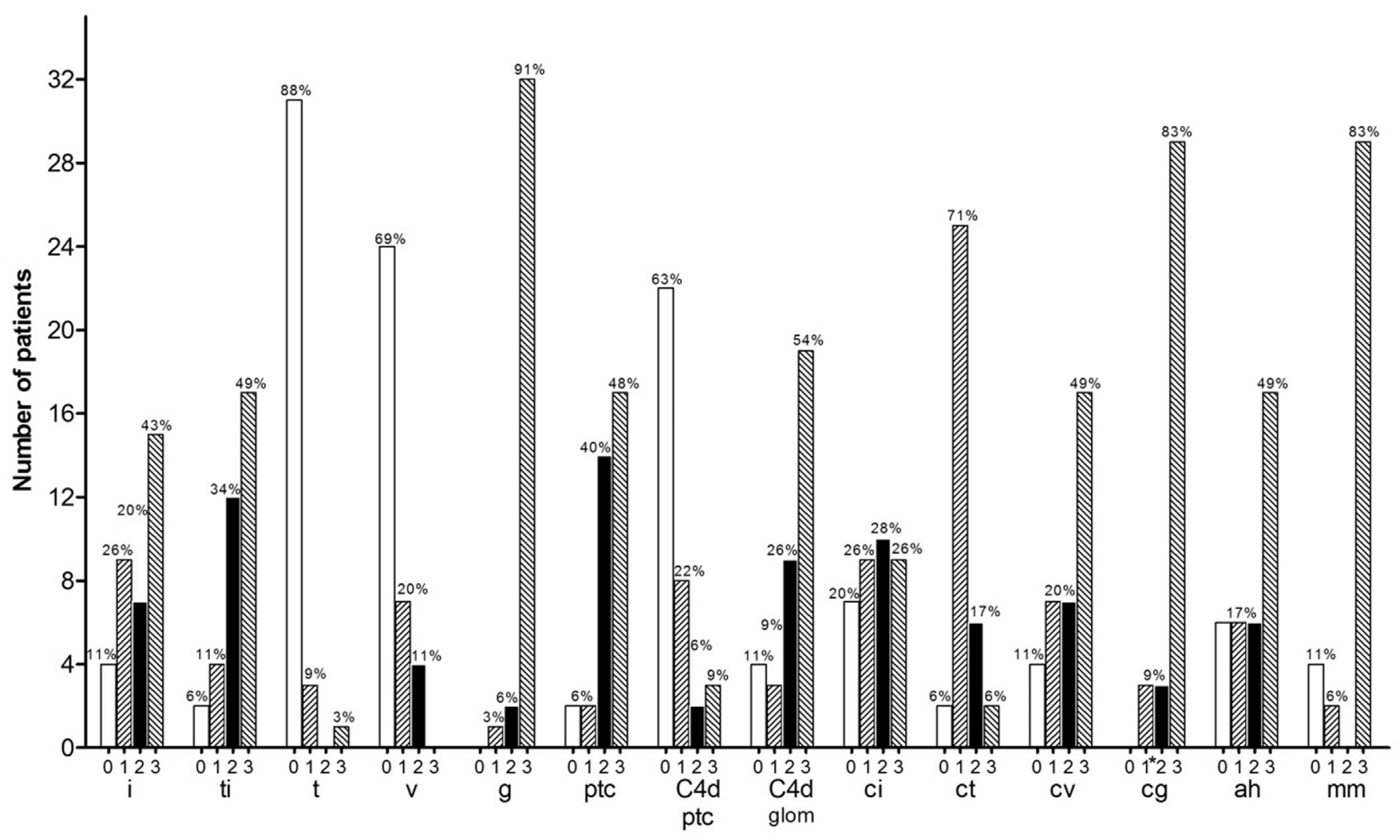

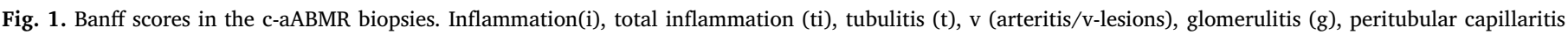

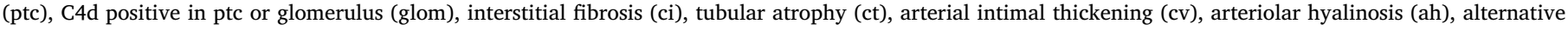
arteriolar hyalinosis score (aah), mesangial thickening (mm).

microvascular inflammation (MVI) was present in all biopsies with a high mean MVI of 5. Arteritis (v-lesion) was present in 11 biopsies (26.8\%). Importantly, minimal tubulitis (t1) was only observed in a minority of cases (31 cases $(75.6 \%)$ with no tubulitis). A combined score (chronicity score) of interstitial fibrosis (ci) + tubular atrophy (ct) + total inflammation (ti) has been identified as a predictor of renal allograft loss in cases with transplant glomerulopathy [16] and was therefore calculated for all cases.

Of note, analysis showed that cases with c-aABMR and arteritis (vlesion) showed a significantly faster decline in graft function in the year before treatment. The decline in function of these patients was $-16.2 \mathrm{~mL} / \mathrm{min} / 1.73 \mathrm{~m}^{2}$ compared $-9.8 \mathrm{~mL} / \mathrm{min} / 1.72 \mathrm{~m}^{2}$ in patients without arteritis $(p<.01)$. However, c-aABMR cases with arteritis did not differ significantly from those without arteritis with respect to all other histomorphological characteristics (data not shown).

In addition, an association was found for total inflammation score $(r=-0.45 p=.007)$ and severity of interstitial fibrosis $(r=-0.38$ $p=.023$ ) with graft function (eGFR) at time of biopsy. None of the Banff lesions related with the degree of proteinuria at time of biopsy. As has been previously published, no relation between the presence of DSA in the serum, Banff scores and graft survival could be found [19].

\subsection{Clinical and histomorphological characteristics in relation to graft survival}

For Kaplan-Meier analysis, cases were divided into low or high histomorphological scores according to a minimal-to-mild score (0-1) or a moderate-to-severe score (2-3). This analysis could not be performed for $\mathrm{cg}, \mathrm{mm}$ and microvascular inflammation (either for glomerulitis, peritubular capillaritis or combined MVI score) as the large majority of cases showed high scores (2 or 3) for these items (Fig. 1). For the remaining lesions higher scores for tubular atrophy (ct), interstitial fibroses (ci), inflammation (i) and total inflammation (ti) were associated with decreased graft survival (Fig. 2). However, interstitial fibrosis significantly correlated with inflammation scores, both inflammation in non-scarred tissue (i) and total inflammation (ti)(respectively $r=0.54=0.001$ and $r=0.50, p=.002$ ). In addition, as expected, the degree of interstitial fibrosis correlated strongly with tubular atrophy $(r=0.64 p<.001)$.

Univariate regression analysis of the Banff lesions showed that total inflammation, interstitial fibrosis and chronicity score were significantly related to the risk for graft failure during follow-up (Table 2). Univariate regression analysis of clinical parameters (Table 1) did not show any variable significantly associated with graft failure except for eGFR at time of biopsy (HR 0.93 per $\mathrm{mL} / \mathrm{min}$ eGFR, $95 \%$ CI $0.88-0.98$, $p=.021)$.

Within the multivariate proportional hazards regression analysis, including the univariate significant Banff lesions and eGFR at time of biopsy, only the degree of interstitial fibrosis (HR 1.9 per score point, $95 \%$ CI 1.2-2.8, $p=.004$ ) was independently associated with graft loss censored for death.

\section{Discussion}

In this study we have analyzed the association of clinical characteristics and Banff lesions in patients with biopsy proven c-aABMR in relation to graft survival.

Most importantly, chronic interstitial fibrosis was the dominant factor associated with graft survival after the diagnosis of c-aABMR. This finding is line with two recent publications which showed that a higher sum chronicity score was associated with inferior graft survival of patients with a diagnosis of transplant glomerulopathy and c-aABMR $[16,20]$.

Previously, proteinuria has been identified in general as a potential 

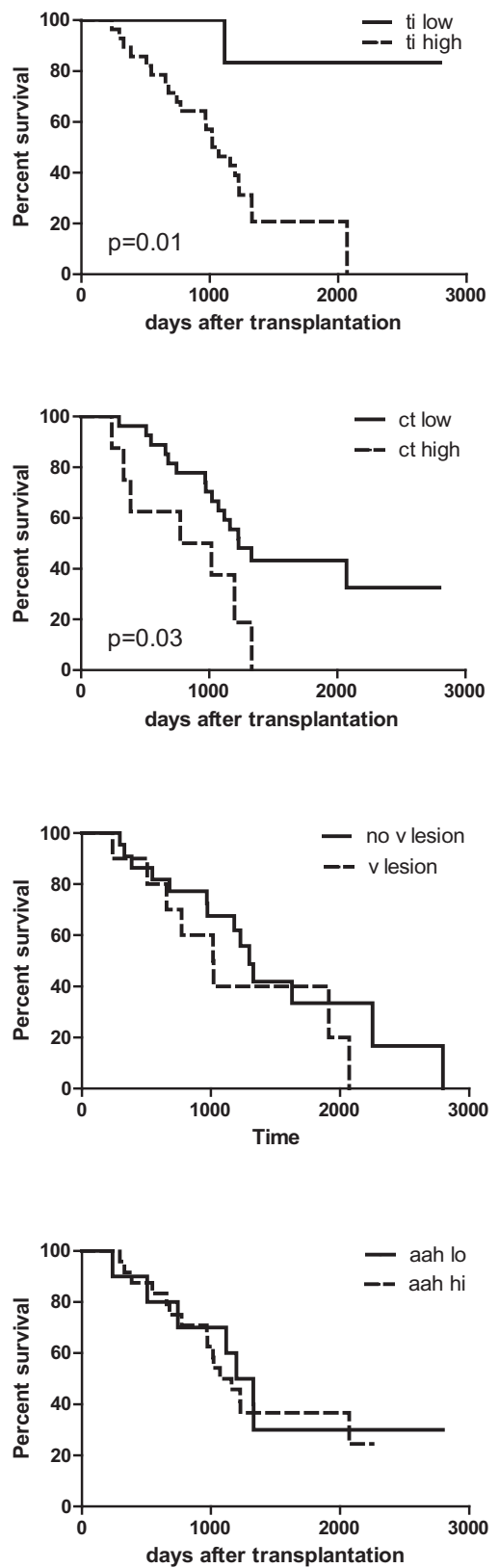
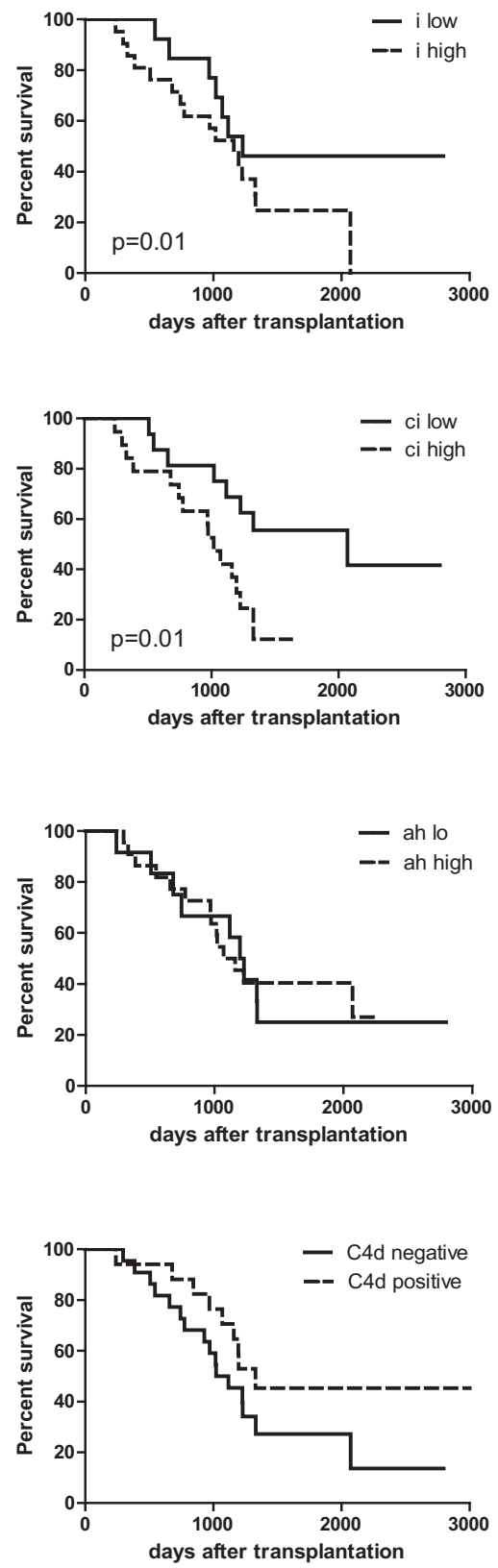

Fig. 2. Kaplan-Meier curves of kidney transplant survival for cases with c-aABMR after the diagnosis and treatment with IVIG/MP. Cases were divided in a low and high score group based on their specific histomorphology scores (low $=$ absent to low score, high = moderate to severe score). Total inflammation (ti) high $n=29$, low $n=6$; inflammation (i), high $n=22$, low $n=13$; tubular atrophy (ct), high $n=8$ and low $n=27$; interstitial fibrosis (ci), high $n=19$, low $n=16$; v-lesions, no $n=24$, present $n=11$; arteriolar hyalinosis (ah), high $n=12$, low $n=23$; alternative arteriolar hyalinosis score (aah), high $n=10$, low $n=25$; C4d score positive $\mathrm{n}=23$, negative $n=17$. $P$-values were calculated by log-rank statistical analysis pairwise over different strata. predictor of poor renal survival. More severe proteinuria might indicate more severe structural damage of the glomerulus and increased inflammation. However, we could not relate the degree of proteinuria with any of the Banff lesions or the risk for allograft loss. This lack of association may be in part caused by the fact that the proteinuria was not normalized for the urine creatinine concentration. However, even if we compared the groups of patients with low proteinuria $(<0.5 \mathrm{~g} / \mathrm{L})$ to high proteinuria $(>0.5 \mathrm{~g} / \mathrm{L})$ we could not find a significant relation with graft survival (data not shown).

As previously suggested by Kahwaji et al., higher MVI scores may be associated with superior response to therapy [15]. Others did find a decrease in MVI score after treatment but without a relation to clinical response [21]. This observation could not be confirmed in our study as the microvascular inflammation in the glomeruli and peritubular capillaries, either separately or combined, was not associated with graft survival. In a recent publication by Viglietti et al., the peritubular capillaritis Banff score was a significant factor in a multivariate model to predict outcome in cases of acute ABMR [22]. However, it should be noted that this result cannot be easily translated to this study as only
$18 \%$ of the cases in their study had chronic allograft glomerulopathy and the average IFTA score was 1 (respectively $100 \%$ and 1.5 in this study). In addition, only a few cases in our study actually had a low MVI which reduces the discriminatory power of this criterion.

As described recently, arteritis can be found in almost half of cases with aABMR (coined antibody-mediated vascular rejection) and is associated with decreased allograft survival as compared to aABMR without arteritis [23]. In our study, arteritis was present in about $1 / 3$ of patients with c-aABMR. Allograft function declined much more rapidly in the cases with arteritis but ultimately did not result in more graft loss. As all cases received steroids and IVIG this may indicate a better response to therapy when v-lesions are present. The origin of the vlesions in the c-aABMR cases is not clear as it cannot be established with certainty whether arteritis represents humoral rejection on the level of the larger vessels or a true T cell-mediated immune response. The latter is not supported by the presence of significant tubulitis in the renal biopsies, but may explain a more favorable response to high dose steroids.

This study has some obvious limitations as it is of retrospective 
Table 2

Median for Banff lesions in renal transplant biopsies with c-aABMR prior to treatment and relation with graft loss after diagnosis.

\begin{tabular}{llll}
\hline & $\begin{array}{l}\text { Median } \\
\text { (range) }\end{array}$ & $\begin{array}{l}\text { Univariate HR } \\
(95 \% \mathrm{CI})^{*}\end{array}$ & p-value \\
\hline ti, total inflammation & $2(0-3)$ & $1.7(0.99-2.9)$ & 0.05 \\
i, interstitial inflammation & $2(0-3)$ & $1.2(0.8-1.8)$ & 0.29 \\
t, tubulitis & $0(0-3)$ & $1.7(0.9-3.2)$ & 0.08 \\
v, arterial inflammation & $0(0-3)$ & $1.1(0.6-1.9)$ & 0.75 \\
g, glomerulitis & $3(0-3)$ & $0.7(0.2-2.1)$ & 0.48 \\
ptc, peritubular capillaritis & $2(0-3)$ & $1.7(0.8-3.3)$ & 0.15 \\
C4d staining peritubular & $0(0-3)$ & $0.8(0.5-1.2)$ & 0.30 \\
$\quad$ capillaries & $3(0-3)$ & $0.8(0.5-1.2)$ & 0.35 \\
C4d staining glomeruli & $2(0-3)$ & $1.8(1.2-2.8)$ & 0.004 \\
ci, interstitial fibrosis & $1(0-3)$ & $1.35(0.7-2.4)$ & 0.32 \\
ct, tubular atrophy & $2(0-3)$ & $1.2(0.8-1.8)$ & 0.35 \\
cv, arterial intimal thickening & $3(0-3)$ & $0.9(0.5-1.6)$ & 0.8 \\
cg, transplant glomerulopathy & $2(0-3)$ & $0.9(0.6-1.2)$ & 0.5 \\
ah, arterial hyalinosis & $3(0-3)$ & $0.9(0.7-1.3)$ & 0.7 \\
AAH, alternative arterial & & & \\
$\quad$ hyalinosis score & $3(0-3)$ & $0.8(0.5-1.1)$ & 0.23 \\
mm, mesangial matrix expansion & $5(1-9)$ & $1.3(1.0-1.6)$ & 0.009 \\
Chronicity score (ci + ct + ti) & & & \\
\hline
\end{tabular}

nature with a relatively small group of patients treated for c-aABMR. However, the cases included are histologically well-defined as c-aABMR and have all received a similar treatment schedule with a largely similar basic immune suppressive regime consisting of a calcineurin inhibitor and mycophenolate mofetil. The uniform treatment schedule in this study is also a limitation as other treatment regimens may show different relations for outcome with clinical variables and Banff scores. In support of our findings, the relation between interstitial fibrosis and graft survival was also observed in 2 studies with a heterogeneous treatment regimen for c-aABMR $[16,20]$. This consistency may not be surprising as the degree of interstitial fibrosis is a commonly found predictor of progressive loss of diseased native kidneys. However, the degree of microvascular inflammation did not predict graft survival, indicating that not inflammation by itself but the resulting interstitial fibrosis is the most important feature of chronic humoral rejection. It is therefore of interest to study more closely the type and functionality of the graft infiltration immune cells in relation to the degree of interstitial fibrosis. Circulating activated immune cells (e.g.NK-cells) poorly correlate with c-aABMR [24] but preliminary data indicate that in particular an influx of activated CD8 T cells in the interstitium is harmful in this respect [25].

The renal biopsies were performed due to a progressive decrease in eGFR which is reflected in the high scores for glomerulopathy and microvascular inflammation. It seems therefore likely that milder or subclinical forms of c-aABMR were not included in this study [26]. Therefore, the associations found between Banff lesions, clinical characteristics and graft survival may not apply for those cases and cases of acute ABMR.

In conclusion, the result of this study shows that the degree of interstitial fibrosis, but not the indicators of inflammation, is associated with graft survival. These findings may be important for risk stratification in clinical trials for c-aABMR and clinical decision making.

\section{Acknowledgements}

The authors are grateful to the department of Virology Erasmus MC for providing serum samples.

\section{Declarations of Competing Interest}

None.

\section{Funding}

This research did not receive any specific grant from funding agencies in the public, commercial, or not-for-profit sectors.

\section{References}

[1] J.D. Pirsch, J. Miller, M.H. Deierhoi, et al., A comparison of tacrolimus (FK506) and cyclosporine for immunosuppression after cadaveric renal transplantation. FK506 kidney transplant study group, Transplant. 63 (1997) 977-983.

[2] R.M. Merion, D.J. White, S. Thiru, et al., Cyclosporine: five years' experience in cadaveric renal transplantation, N. Engl. J. Med. 310 (1984) 148-154.

[3] K.E. Lamb, S. Lodhi, H.U. Meier-Kriesche, Long-term renal allograft survival in the United States: a critical reappraisal, Am. J. Transplant. 11 (2011) 450-462.

[4] T. Fehr, A. Gaspert, Antibody-mediated kidney allograft rejection: therapeutic options and their experimental rationale, Transpl. Int. 25 (2012) 623-632.

[5] G. Bartel, E. Schwaiger, G.A. Bohmig, Prevention and treatment of alloantibodymediated kidney transplant rejection, Transpl. Int. 24 (2011) 1142-1155.

[6] L.C. Racusen, K. Solez, R.B. Colvin, et al., The Banff 97 working classification of renal allograft pathology, Kidney Int. 55 (1999) 713-723.

[7] L.C. Racusen, R.B. Colvin, K. Solez, et al., Antibody-mediated rejection criteria - an addition to the Banff 97 classification of renal allograft rejection, Am. J. Transplant. 3 (2003) 708-714.

[8] F.G. Cosio, J.P. Grande, H. Wadei, et al., Predicting subsequent decline in kidney allograft function from early surveillance biopsies, Am. J. Transplant. 5 (2005) 2464-2472.

[9] F.G. Cosio, J.M. Gloor, S. Sethi, et al., Transplant glomerulopathy, Am. J. Transplant. 8 (2008) 492-496.

[10] J.M. Gloor, S. Sethi, M.D. Stegall, et al., Transplant glomerulopathy: subclinical incidence and association with alloantibody, Am. J. Transplant. 7 (2007) 2124-2132.

[11] R.K. Maryniak, M.R. First, M.A. Weiss, Transplant glomerulopathy: evolution of morphologically distinct changes, Kidney Int. 27 (1985) 799-806.

[12] F. Eskandary, H. Regele, L. Baumann, et al., A randomized trial of Bortezomib in late antibody-mediated kidney transplant rejection, J. Am. Soc. Nephrol. 29 (2018) 591-605.

[13] R.A. Montgomery, A. Loupy, D.L. Segev, Antibody-mediated rejection: new approaches in prevention and management, Am. J. Transplant. 18 (Suppl. 3) (2018) $3-17$.

[14] F. Moreso, M. Crespo, J.C. Ruiz, et al., Treatment of chronic antibody mediated rejection with intravenous immunoglobulins and rituximab: a multicenter, prospective, randomized, double-blind clinical trial, Am. J. Transplant. 18 (2018) 927-935.

[15] J. Kahwaji, R. Najjar, D. Kancherla, et al., Histopathologic features of transplant glomerulopathy associated with response to therapy with intravenous immune globulin and rituximab, Clin. Transpl. 28 (2014) 546-553.

[16] P. Patri, S.V. Seshan, M. Matignon, et al., Development and validation of a prognostic index for allograft outcome in kidney recipients with transplant glomerulopathy, Kidney Int. 89 (2016) 450-458.

[17] A. Loupy, M. Haas, K. Solez, et al., The Banff 2015 kidney meeting report: current challenges in rejection classification and prospects for adopting molecular pathology, Am. J. Transplant. 17 (2017) 28-41.

[18] K.A. Sablik, M.C. Clahsen-van Groningen, C.W.N. Looman, et al., Treatment with intravenous immunoglobulins and methylprednisolone may significantly decrease loss of renal function in chronic-active antibody-mediated rejection, BMC Nephrol. 20 (2019) 218.

[19] K.A. Sablik, M.C. Clahsen-van Groningen, C.W.N. Looman, et al., Chronic-active antibody-mediated rejection with or without donor-specific antibodies has similar histomorphology and clinical outcome - a retrospective study, Transpl. Int. 31 (2018) 900-908.

[20] S. Parajuli, E. Joachim, S. Alagusundaramoorthy, et al., Subclinical antibody mediated rejection after kidney transplantation: treatment outcomes, Transplantation (Feb 4 2019), https://doi.org/10.1097/TP.0000000000002566 (Epub ahead of print).

[21] A. Mella, E. Gallo, M. Messina, et al., Treatment with plasmapheresis, immunoglobulins and rituximab for chronic-active antibody-mediated rejection in kidney transplantation: clinical, immunological and pathological results, World J. Transplant. 8 (2018) 178-187.

[22] D. Viglietti, A. Loupy, O. Aubert, et al., Dynamic prognostic score to predict kidney allograft survival in patients with antibody-mediated rejection, J. Am. Soc. Nephrol. 29 (2018) 606-619.

[23] C. Lefaucheur, A. Loupy, D. Vernerey, et al., Antibody-mediated vascular rejection of kidney allografts: a population-based study, Lancet 381 (2013) 313-319.

[24] K.A. Sablik, N.H.R. Litjens, M. Klepper, et al., Increased CD16 expression on NK cells is indicative of antibody-dependent cell-mediated cytotoxicity in chronic-active antibody-mediated rejection, Transpl. Immunol. 54 (2019) 52-58.

[25] K.J.K. Sablik, Groningen MClahsen-van, M. Betjes, Increased number of Intragraft FoxP3 + T cells is strongly correlated with decreased graft survival in chronic active antibody mediated rejection [abstract], Am. J. Transplant. 19 (Suppl. 3) (2019).

[26] A. Loupy, D. Vernerey, C. Tinel, et al., Subclinical rejection phenotypes at 1 year post-transplant and outcome of kidney allografts, J. Am. Soc. Nephrol. 26 (2015) 1721-1731. 